\title{
Neonatal outcome after pregnancy complicated by abnormal velocity waveforms in the umbilical
} artery

\author{
Maura McDonnell, Vicente Serra-Serra, Geraldine Gaffney, Christopher W G Redman, \\ Peter L Hope
}

\begin{abstract}
The neonatal outcome of 61 infants born after pregnancies complicated by absent or reversed end diastolic flow velocities (AREDFV) in the fetal umbilical artery was compared with that of 61 controls matched for gestational age born after high risk pregnancies with documented forward end diastolic flow velocities (EDFV). The AREDFV group was significantly more growth retarded, had lower platelet counts at birth, and were more likely to become significantly thrombocytopenic in the first week after birth. Owing to concerns about the possible increased risk of necrotising enterocolitis in newborn infants after AREDFV, this group was started on enteral feeds later and was more likely to receive parenteral nutrition than the EDFV group. Seven infants with AREDFV and one control infant developed necrotising enterocolitis. (Arch Dis Child 1994; 70: F84-F89)
\end{abstract}

The modern obstetric assessment of a high risk pregnancy often includes Doppler ultrasonography of the circulation of the fetus. End diastolic flow velocities (EDFV) in the umbilical artery of the growth retarded fetus may be reduced, absent, or even reversed as a consequence of increased placental vascular resistance. ${ }^{12}$ Similar changes may occur in the fetal thoracic aorta and may be associated with the redistribution of fetal cardiac output to maintain cerebral metabolism and growth when fetal oxygen and substrate supply are limited by placental insufficiency. ${ }^{3}$

Two small studies have shown high neonatal mortality and morbidity in infants born after pregnancies complicated by absent or reversed end diastolic flow velocities (AREDFV) in the umbilical artery ${ }^{4}$ or fetal thoracic aorta. ${ }^{3}$ These two studies showed an excess of necrotising enterocolitis in the infants with AREDFV. It has been suggested that the redistribution of fetal cardiac output to achieve 'brain-sparing' may lead to impaired gut perfusion in utero and predispose to necrotising enterocolitis in the neonatal period. If this predisposition to necrotising enterocolitis is real, then there may be a rationale for delayed enteral feeding in this high risk group with AREDFV because necrotising enterocolitis is rare in the absence of intestinal substrate for bacterial growth.

This paper reports the neonatal morbidity and mortality of a large group of infants, born to mothers cared for by a single high risk pregnancy service over a seven year period, who were known to have AREDFV. Neonatal variables were compared with those of controls matched for gestational age and sex who had forward EDFV on umbilical cord Doppler studies and who were cared for by the same service.

\section{Patients and methods}

DOPPLER METHODS

Since 1985 the assessment of high risk pregnancies referred to our hospital has included Doppler studies of umbilical artery velocity waveforms. Thirty three pregnancies were assessed using a $4 \mathrm{MHz}$ continuous wave device with a $150 \mathrm{~Hz}$ high pass filter, which is suboptimal for the accurate detection of absent $\mathrm{EDFV}$, and were therefore excluded from this analysis. The rest of the assessments were performed using either $4 \mathrm{MHz}$ continuous wave Doppler scanner with a $0 \mathrm{~Hz}$ wall filter (Angioscan, Appleton, Chilworth, UK) or a $3.5 \mathrm{MHz}$ duplex Doppler scanner with a $50 \mathrm{~Hz}$ wall filter (Ultramark 5, ATL, Washington, USA). Doppler waveforms were analysed only if a clear umbilical vein flow pattern was seen on the record.

During the period covered by this paper Doppler studies were used to define a high risk population which needed full assessment by other techniques. The presence of AREDFV was not regarded as an indication for immediate delivery, the timing of which was determined on clinical and cardiotocographic grounds using computerised cardiotocographic analysis. ${ }^{5}$

\section{PATIENTS}

Ninety two fetuses with AREDFV were identified. There were 14 intrauterine deaths. Five infants were outborn and one was transferred 
Table 1 Clinical details of the mothers and their pregnancies

\begin{tabular}{lll}
\hline Clinical details & AREDFV & Controls \\
\hline No of infants (mothers) & $61(61)$ & $61(60)$ \\
Median (range) maternal age (years) & $28(18-41)$ & $29(16-39)$ \\
No (\%) of primipara & $42(69)$ & $36(60)$ \\
No (\%) with pre-eclampsia & $38(62)$ & $44(73)$ \\
No (\%) requiring caesarean section & $59(97)$ & $56(93)$ \\
No (\%) treated with antenatal & $16(26)$ & $12(20)$ \\
\begin{tabular}{l} 
steroids \\
\hline
\end{tabular}
\end{tabular}

No significant differences between the groups.

back to the referring hospital on the first day of life. Two of these six died, one with multiple congenital abnormalities. One inborn infant, a twin of birth weight $400 \mathrm{~g}$, was considered non-viable and died without admission to the neonatal unit. Two infants were still on the neonatal unit at the time of analysis and their outcome was uncertain, and the notes of two other infants were missing. Of the remaining 67 infants it was impossible to match six for gestational age and sex with an infant with forward diastolic flow.

Each of the remaining 61 infants of mothers whose pregnancy was complicated by AREDFV was retrospectively paired with a control infant born to a mother cared for by the same high risk pregnancy service who had documented forward EDFV in the umbilical artery. Controls were matched for sex and gestational age within one week. When more

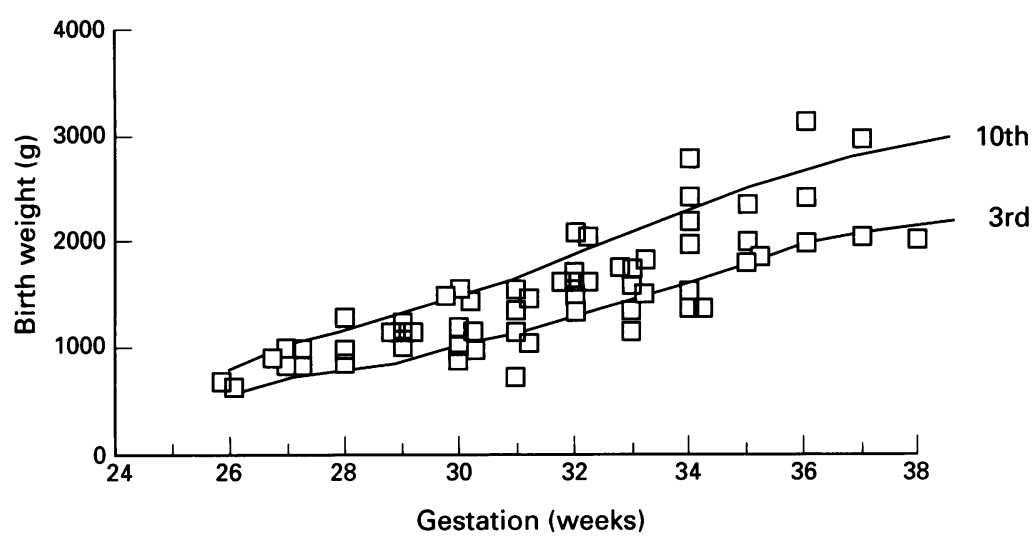

Figure 1 Gestational age and birth weight of the 61 control neonates (3rd and 10th centiles derived from the data of Yudkin et $\mathrm{al}^{6}$ ).

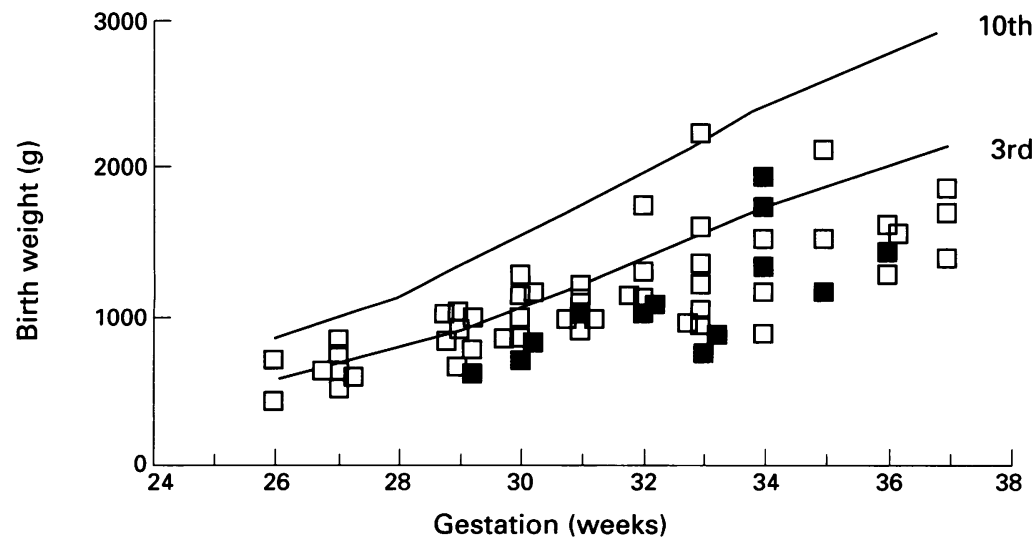

Figure 2 Gestational age and birth weight of the 61 neonates born after pregnancies complicated by AREDFV in the umbilical artery. Closed symbols represent the neonates with thrombocytopenia $\left(<100 \times 10^{9} /\right)$ on the initial platelet count (3rd and 10th centiles derived from the data of Yudkin $\mathrm{et}^{6} \mathrm{al}^{6}$ ). than one suitable control was available, the infant with the nearest date of birth to the index case was selected.

\section{STUDY DESIGN AND DEFINITIONS}

Cases were identified by the obstetric team and matched controls were retrospectively allocated by a paediatrician. Notes of the patients and controls were retrospectively reviewed with respect to a range of neonatal variables. Neither the doctor caring for the neonate nor the paediatrician reviewing the case notes was blind to the antenatal Doppler studies.

Cord umbilical arterial samples were taken by a midwife from a length of cord which was double clamped at delivery. Initial haematological results were obtained from routine laboratory analysis of a sample taken from the infant on admission to the neonatal unit.

Infants were classified as hypotensive if the doctor treating them had used colloid infusions or inotropes to treat hypotension in the first few days of life. Our neonatal unit policy is to treat all asymptomatic neonates with a mean arterial blood pressure less than $30 \mathrm{~mm} \mathrm{Hg}$ (measured with an indwelling arterial catheter) and infants with poor peripheral perfusion, especially if accompanied by systemic acidosis or oliguria.

Infants requiring mechanical ventilation had hourly nursing records of peak and mean airway pressures, which were used to find the maximum values.

Necrotising enterocolitis was diagnosed in infants who became systemically unwell with abdominal distension, who also had feed intolerance, or rectal bleeding, or both. In all instances the doctor caring for the infant stopped enteral feeds and started antibiotic treatment and parenteral nutrition.

Statistical comparisons between cases and matched controls were made using the $\chi^{2}$ test or Fisher's exact test, or the Wilcoxon signed rank test for continuous variables. The MannWhitney test was used for unpaired comparisons.

\section{Results}

There was no significant difference between pregnancies complicated by AREDFV and the matched controls with respect to maternal age, parity, antenatal steroid treatment, or mode of delivery (table 1). Most of the mothers had a diagnosis of pre-eclampsia and almost all were delivered by caesarean section. Table 2 gives the clinical details of the 61 infants with AREDFV and their controls matched for gestational age and sex. The cases were markedly more growth retarded; 47 (77\%) were less than the third centile compared with $15(25 \%)$ of the matched controls. Figures 1 and 2 show the gestational age and birthweight distributions of cases and controls.

There was no significant difference in cord arterial $\mathrm{pH}$ between the groups: the median cord artery $\mathrm{pH}$ was $7 \cdot 23$ in cases and $7 \cdot 25$ in controls. 
About half of each group required mechanical ventilation, usually for hyaline membrane disease. There was no difference between the groups with respect to the duration of mechanical ventilation, the maximum inspired oxygen concentration, maximum peak or maximum mean airway pressure. Despite the fact that this was a high risk group of infants $(39(32 \%)$ of the total group of 122 cases and controls were $<1000 \mathrm{~g}$ birth weight), parenchymal cerebral lesions and neonatal death were rare. Only four infants had intraparenchymal cerebral haemorrhage or cystic periventricular leukomalacia, and only three died.
Table 3 gives the initial haematological results for the two groups. The initial packed cell volumes were similar, but there were highly significant differences in the white blood cell and platelet counts. The initial platelet counts were significantly lower in the cases, 12 $(20 \%)$ of whom had an initial platelet count $<100 \times 10 \mathrm{~g}^{9} / 1$ compared with none of the controls. The platelet count often decreased during the first week of life in the two groups. Minimum platelet counts of $<100 \times 10^{9} / 1$ were recorded in $29(48 \%)$ cases and five $(8 \%)$ controls at a median of 5 (range 1-11) days of age. Figure 2 shows that thrombocytopenia at birth occurred over a wide range of gestational

Table 2 Clinical details of the neonates

\begin{tabular}{lccc}
\hline Clinical details & AREDFV $(n=61)$ & Controls $(n=61)$ & $p$ Value \\
\hline Median (range) birth weight (g) & $1014(425-2191)$ & $1483(642-3206)$ & $<0 \cdot 0001$ \\
Median (range) gestational age (weeks) & $32(26-37)$ & $32(26-38)$ & $\mathrm{NS}$ \\
No (\%) boys & $30(49)$ & $30(49)$ & $\mathrm{NS}$ \\
No (\%) with birth weight below third centile & $47(77)$ & $15(25)$ & $<0 \cdot 00001$ \\
Median (range) umbilical cord artery pH & $7 \cdot 23(6 \cdot 88-7 \cdot 32)^{\star}$ & $7 \cdot 25(6 \cdot 84-7 \cdot 34) \dagger$ & $\mathrm{NS}$ \\
No (\%) requiring mechanical ventilation & $27(44)$ & $25(41)$ & $\mathrm{NS}$ \\
Median (range) maximum peak airway pressure (cm $\left.\mathrm{H}_{2} \mathrm{O}\right)$ & $20(12-38)$ & $20(15-33)$ & $\mathrm{NS}$ \\
Median (range) duration of mechanical ventilation $($ days) & $7(1-48)$ & $6(1-32)$ & $\mathrm{NS}$ \\
No (\%) with hypotension (MABP<30 mm Hg) & $11(18)$ & $11(18)$ & $\mathrm{NS}$ \\
No (\%) with inotrope treatment & $6(10)$ & $5(8)$ & $\mathrm{NS}$ \\
Median (range) age first enteral feeds (days) & $4(1-19)$ & $2(1-18)$ & $<0 \cdot 05$ \\
No (\%) receiving parenteral nutrition & $36(59)$ & $12(20)$ & $<0 \cdot 0001$ \\
No (\%) with necrotising enterocolitis & $7(11)$ & $1(2)$ & $0 \cdot 07$ \\
No (\%) with parenchymal brain lesions & $3(5)$ & $1(2)$ & $\mathrm{NS}$ \\
No (\%) of neonatal deaths & $3(5)$ & & $\mathrm{NS}$ \\
\hline
\end{tabular}

$\mathrm{MABP}=$ mean arterial blood pressure. ${ }^{\star} n=47 . \dagger \mathrm{f}=50$.

Table 3 Haematological details of the neonates

\begin{tabular}{lccl}
\hline Haematological details & Cases $(n=61)$ & Controls $(n=61)$ & $p$ Value \\
\hline Median (range) initial packed cell volume & $0 \cdot 56(0 \cdot 33-0 \cdot 75)$ & $0 \cdot 56(0 \cdot 44-0 \cdot 72)$ & NS \\
Median (range) initial WBC count $\left(\times 10^{9} /\right)$ & $17(6-53)$ & $11(5-38)$ & $<0 \cdot 0005$ \\
Median (range) initial platelet count $\left(\times 10^{9} /\right)$ & $152(33-232)$ & $209(111-437)$ & $<0 \cdot 0001$ \\
No $(\%)$ with initial platelet count $<100 \times 10^{9} / 1$ & $12(20)$ & 0 & $<0 \cdot 001$ \\
No (\%) with minimum platelet count $<100 \times 10^{9} / 1$ & $29(48)$ & $3(8)$ & $<0.00001$ \\
No (\%) with minimum platelet count $<50 \times 10^{9} / 1$ & $15(25)$ & $6(1-28)$ & $<0.005$ \\
Median (range) age at minimum platelet count (days) & $5(1-11)$ & NS \\
\hline
\end{tabular}

One packed cell volume, two platelet counts, and three WBC counts were missing. WBC=white blood cell.

Table 4 Clinical details of infants with necrotising enterocolitis

\begin{tabular}{|c|c|c|c|c|c|c|c|}
\hline $\begin{array}{l}\text { Gestational } \\
\text { age (weeks) }\end{array}$ & $\begin{array}{l}\text { Birth weight } \\
\text { (g) }\end{array}$ & $\begin{array}{l}\text { Abdominal } \\
\text { distension }\end{array}$ & $\begin{array}{l}\text { Feed } \\
\text { intolerance }\end{array}$ & $\begin{array}{l}\text { Faecal } \\
\text { blood }\end{array}$ & $\begin{array}{l}\text { Suspicious } \\
\text { radiology }\end{array}$ & $\begin{array}{l}\text { Definite } \\
\text { radiology }\end{array}$ & $\begin{array}{l}\text { Operation } \\
\text { performed }\end{array}$ \\
\hline \multicolumn{8}{|l|}{ Cases } \\
\hline 27 & 732 & + & + & + & + & & \\
\hline 34 & 864 & + & + & & & & \\
\hline 30 & 1137 & + & + & + & + & & \\
\hline 35 & 1142 & + & + & + & + & & \\
\hline 27 & 594 & + & + & + & + & & \\
\hline 33 & 717 & + & & + & & + & + \\
\hline 30 & 830 & + & + & + & & + & \\
\hline \multicolumn{8}{|l|}{ Control } \\
\hline 30 & 1240 & + & + & + & & + & + \\
\hline
\end{tabular}

$+=$ Present.

Table 5 Comparison of infants with absent $(A)$ and reversed $(R)$ EDFV

\begin{tabular}{|c|c|c|c|}
\hline & $A E D F V(n=46)$ & $R E D F V(n=15)$ & p Value \\
\hline $\begin{array}{l}\text { Median (range) birth weight (g) } \\
\text { Median (range) gestational age (weeks) } \\
\text { No (\%) with birth weight less than third centile } \\
\text { Median (range) umbilical cord artery pH } \\
\text { No (\%) requiring mechanical ventilation } \\
\text { Median (range) maximum peak airway pressure }\left(\mathrm{cm} \mathrm{H}_{2} \mathrm{O}\right) \\
\text { Median (range) duration of mechanical ventilation (days) } \\
\text { No }(\%) \text { with hypotension (MABP }<30 \text { mm } \mathrm{Hg}) \\
\text { Median (range) age first enteral feeds (days) } \\
\text { No }(\%) \text { receiving parenteral nutrition } \\
\text { No }(\%) \text { with necrotising enterocolitis } \\
\text { Median (range) initial packed cell volume } \\
\text { Median (range) initial WBC count }\left(\times 10^{9} / 1\right) \\
\text { Median (range) initial platelets }\left(\times 10^{9} /\right) \\
\text { No }(\%) \text { with minimum platelet count }<50\left(\times 10^{9} / 1\right)\end{array}$ & $\begin{array}{l}1098(425-2191) \\
33(26-37) \\
36(78) \\
7 \cdot 25(6 \cdot 88-7 \cdot 32)^{\star} \\
18(39) \\
20(15-32) \\
8(1-48) \\
7(15) \\
3(1-15) \\
22(48) \\
5(11) \\
0 \cdot 50(0 \cdot 33-0 \cdot 67) \\
16(6-53) \\
152(51-232) \\
7(15)\end{array}$ & $\begin{array}{l}975(585-1898) \\
30(26-34) \\
11(71) \\
7 \cdot 12(6 \cdot 92-7 \cdot 28) \dagger \\
9(60) \\
20(12-38) \\
9(1-38) \\
4(27) \\
8(2-19) \\
13(87) \\
2(13) \\
0 \cdot 57(0 \cdot 42-0 \cdot 75) \\
20(7-42) \\
134(33-217) \\
8(53)\end{array}$ & $\begin{array}{l}\text { NS } \\
<0.05 \\
\text { NS } \\
<0.01 \\
\text { NS } \\
\text { NS } \\
\text { NS } \\
\text { NS } \\
<0.01 \\
<0.05 \\
\text { NS } \\
<0.05 \\
\text { NS } \\
\text { NS } \\
<0.01\end{array}$ \\
\hline
\end{tabular}

$\mathrm{MABP}=$ mean arterial blood pressure; $\mathrm{WBC}=$ white blood cell. ${ }^{\star} \mathrm{n}=34 . \mathrm{fn}=13$. 
ages, but tended to affect the most growth retarded infants.

Pulmonary haemorrhage occurred in only one case and no controls, and germinal layer or intraventricular haemorrhage occurred in eight infants in each group.

Enteral feeds were started later in infants with AREDFV, who were also much more likely to receive parenteral nutrition (table 2). Seven cases and only one control had a clinical diagnosis of necrotising enterocolitis (details in table 4), a difference which is not statistically significant. The overall incidence of necrotising enterocolitis was low. Only three cases from the whole group had unequivocal intramural gas, only two required an operation (for late strictures), and there was no necrotising enterocolitis associated mortality.

One of the seven infants with AREDFV who developed necrotising enterocolitis had Down's syndrome and congenital heart disease. The data were reanalysed excluding this infant, two other cases with Down's syndrome who did not develop necrotising enterocolitis, and their matched controls. This analysis of infants with normal karyotypes showed necrotising enterocolitis in six $(10 \%)$ of 58 infants after AREDFV and none in 58 controls, which is a significant difference $(p=0.027)$.

Table 5 gives the differences within the 61 cases with AREDFV between the group of 46 infants who had shown absent EDFV in utero and the group of 15 infants with reversed EDFV. The 15 patients with reversed EDFV were of lower gestational age but similar birth weight. As a group they were more acidotic at delivery, but had no greater incidence or severity of respiratory disease or major complications. Although their initial platelet counts were no different from the group with absent but not reversed EDFV, they were more likely to develop thrombocytopenia postnatally. Feeds were introduced significantly later in the reversed EDFV group.

\section{Discussion}

Two previous studies have suggested a possible association between fetal AREDFV and neonatal necrotising enterocolitis. Hackett et al compared 26 high risk neonates with AREDFV with 20 unmatched infants with forward EDFV using Doppler apparatus with a $150 \mathrm{~Hz}$ filter. ${ }^{3}$ Malcolm et al used a system with a $100 \mathrm{~Hz}$ filter and studied 15 neonates with AREDFV and 15 controls matched for birth weight. ${ }^{4}$ These two studies were small and used filters suboptimal for the accurate identification of true AREDFV. Nevertheless, because necrotising enterocolitis is a life threatening illness which is rare before the start of enteral feeds, these reports have caused some units to delay the introduction of enteral feeds in neonates where AREDFV has been documented in utero. Such a policy denies these high risk infants the benefits of breast milk and exposes them to total parenteral nutrition and the associated risks of sepsis, poor weight gain, and hepatic, pulmonary, or cerebral sequelae.
An earlier analysis of the data from our unit, ${ }^{7}$ looking at the outcome of 21 neonates born after AREDFV in the umbilical artery, suggested there was no excess of necrotising enterocolitis in that small sample. As the result of a subsequent clinical impression of reduced feed tolerance after AREDFV, we carried out this further retrospective review of our own experience to see whether the association of AREDFV with necrotising enterocolitis and other neonatal morbidity was confirmed in a larger population of high risk neonates studied with Doppler equipment incorporating 0 or 50 $\mathrm{Hz}$ filters. The obstetric management and outcome of these pregnancies will be discussed in detail elsewhere.

The original observations of Hackett et al suggested a higher incidence of necrotising enterocolitis, bleeding problems, and death in neonates who had documented AREDFV in the thoracic aorta in utero. ${ }^{3}$ Our study considers the outcome of fetuses with AREDFV in the umbilical artery, a group which may be expected to be even more severely compromised as diastolic flow velocity changes in the aorta are thought to precede those in the umbilical artery. ${ }^{8}$ The AREDFV group in our study had birth weights and haematological indices suggestive of greater intrauterine compromise. We did not show such a clear increase in neonatal morbidity as Hackett et al reported, ${ }^{3}$ though our results with respect to necrotising enterocolitis may well be confounded by differences in the management of the AREDFV group.

Fourteen $(15 \%)$ of the 92 fetuses with AREDFV died in utero. Of the liveborn infants, seven $(9 \%)$ of 78 died before discharge from hospital, three outside our unit. Our results may underestimate the problems associated with AREDFV as six infants were excluded because suitable gestation matched controls could not be identified. All six were $<1000 \mathrm{~g}$ birth weight, one developed necrotising enterocolitis, but all survived. Data from all 61 neonates with AREDFV who survived to receive intensive care on our unit, and could be matched for gestation, show clearly that this group was severely growth retarded (fig 2).

Only four (3\%) of 122 cases and controls in this comparison died before discharge from hospital and only three (2\%) of 122 had parenchymal brain lesions detected by regular cranial ultrasound, so this study lacked the power to determine the effect of AREDFV on such rare outcomes.

The timing of delivery was never determined by Doppler data but was decided on the basis of a biophysical profile, computerised cardiotocographic analysis, maternal status, and discussion between the high risk pregnancy service and the neonatologists. Although the cord arterial $\mathrm{pH}$ was often slightly lower than would be expected for infants born by caesarean section without labour, this policy led to most of the cases and controls being delivered before severe fetal acidosis supervened.

The AREDFV group did not require any more ventilatory assistance than controls, as might be expected if the frequency and severity 
of surfactant deficiency are determined more by gestation than birth weight. Although severe intrauterine hypoxia can lead to impaired myocardial contractility postnatally, there was no excess of early hypotension in the study group. The AREDFV group therefore did not seem disadvantaged in terms of condition at birth or cardiorespiratory status in the neonatal period.

There was nevertheless an excess of necrotising enterocolitis in the AREDFV group. In contrast with previous reports, this excess was not statistically significant. It could be argued that the infants with Down's syndrome and their controls should be excluded from the study, in which instance statistical significance is achieved. The fact that the AREDFV group started enteral feeds later in life, and were much more likely to receive parenteral nutrition, may be partly due to lower birth weight. We are aware that it was also due to a reluctance to start early enteral feeds after AREDFV in the light of previous reports. Towards the end of the study period several infants of $<1000 \mathrm{~g}$ birth weight born after AREDFV received elective parenteral nutrition for the first week of life on the basis of antenatal Doppler studies. If delayed enteral feeding is indeed protective against necrotising enterocolitis (that is, if the advantage of limiting intestinal substrate for bacterial growth outweighs the risk of delayed introduction of breast milk), then elective total parenteral nutrition of infants after AREDFV may have reduced the incidence of necrotising enterocolitis in that group. The fact that we still see a tendency towards more necrotising enterocolitis after AREDFV is therefore compatible with the hypothesis of Hackett et $a l^{3}$ that AREDFV may lead to fetal intestinal ischaemia and thus predispose to neonatal necrotising enterocolitis.

The doctors responsible for the care of these neonates were aware of the fetal Doppler results, so there was a potential source of bias in that necrotising enterocolitis may have been more readily diagnosed in the AREDFV group. We attempted to minimise this effect by including as cases of necrotising enterocolitis all infants whose notes on retrospective review contained reports of systemic illness with abdominal distension, and increased aspirates or bloody stools (table 4). This definition includes infants who would be described by other workers as 'suspicious' cases of necrotising enterocolitis. Only three $(2 \%)$ of 122 cases and controls had unequivocal necrotising enterocolitis with characteristic radiological changes and the two infants requiring an operation did well after the late resection of strictures. The overall mortality from necrotising enterocolitis in this study was zero, and morbidity was low.

The study of Malcolm et al reported changes in haematological indices in their AREDFV group, which did not reach statistical significance. ${ }^{4}$ This study shows that platelet counts at birth are significantly lower after AREDFV and this effect is not confined to the infants with the lowest birth weights or gestational age (fig 2). The AREDFV group was also much more likely to develop significant thrombocytopenia postnatally. The low platelet count at birth in infants who have been severely compromised in utero is well recognised and has been attributed to the diversion of pluripotent stem cells into the production of red cell precursors during conditions of hypoxic stress. $^{9}$ The further postnatal decrease in the platelet count to a nadir around 5 days of age is often seen, and in the absence of sepsis is presumably due to an altered balance in platelet production and consumption in early postnatal life. We did not see an excess of intracranial or overt pulmonary haemorrhage as a consequence of thrombocytopenia, but we were not observing the natural history of this complication because platelet transfusions were given to severely thrombocytopenic infants.

The increased white blood cell count seen on initial full blood counts from the AREDFV group was an interesting finding which we were not anticipating. Differential white blood cell counts and normoblast counts were therefore not obtained on many infants, so the reported total white blood cell counts are difficult to interpret. They probably represent increased numbers of red cell precursors in the most compromised fetuses.

The small subset $(15 / 61 ; 25 \%)$ of the AREDFV group who had reversed EDFV required delivery on cardiotocographic and clinical grounds at a significantly lower gestational age than the group with AEDFV. Haematological indices suggested greater intrauterine compromise in the group with reversed $\mathrm{EDFV}$, and cord arterial $\mathrm{pH}$ values were significantly lower, implying a degree of fetal decompensation.

Although the Doppler data used in this study were all recorded prospectively, the neonatal data were collected retrospectively. As the Doppler results were not withheld from doctors there are clearly sources of potential bias, which we sought to minimise in the case of 'qualitative' outcomes such as necrotising enterocolitis. 'Hard' data such as platelet counts were only susceptible to bias in the selection of the matched controls, which was not a problem because there was usually only potential control of comparable sex and birth weight with documented Doppler data and antenatal care by the same high risk pregnancy service. If there was more than one suitable control the infant with the nearest birth date was used.

These data confirm previous reports suggesting an increased incidence of neonatal problems after fetal compromise as determined by abnormal fetal blood flow velocity waveforms. Infants born after pregnancies complicated by AREDFV were more growth retarded than controls matched for gestational age, and more likely to be thrombocytopenic. Although necrotising enterocolitis was not a major cause of mortality or morbidity in this group, there was a tendency towards a higher incidence in the AREDFV group despite delayed enteral feeding. 
We thank Ravi Chandran, Tansy Cheston, Karen Hughes, Regina Imhof, June Jennings, Vibeke Mannion, Nicky Manning, and Robert Pattinson who performed the fetal assessments, and the medical and nursing staff of the neonatal unit.

1 Trudinger BJ, Giles WB, Cook CM, Bombardieri J, Collins L. Fetal umbilical artery flow velocity waveforms and placental resistance: clinical significance. $\mathrm{Br} \mathcal{F}$ Obstet Gynaecol 1985; 92: 23-30.

2 Brar HS, Platt LD, Reverse end-diastolic flow velocity on umbilical artery velocimetry in high-risk pregnancies: an ominous finding with adverse pregnancy outcome. $\operatorname{Am} \mathcal{f}$ Obstet Gynecol 1988; 159: 559-61.

3 Hackett GA, Campbell S, Gamsu H, Cohen-Overbeek T, Pearce JMF. Doppler studies in the growth retarded fetus and prediction of neonatal necrotising enterocolitis, and prediction of neonatal necrotising enterocolitis, haemo.

4 Malcolm G, Ellwood D, Devonald K, Beilby R, HendersonSmart D. Absent or reversed end diastolic flow velocity in the umbilical artery and necrotising enterocolitis. Arch Dis Child 1991; 66: 805-7.

5 Dawes GS, Redman CWG, Smith JH. Improvements in the registration and analysis of fetal heart rate records at the bedside. Br f Obstet Gynaecol 1985; 92: 317-25.

6 Yudkin PL, Aboualfa M, Eyre JA, Redman CWG, Wilkinson AR. New birthweight and head circumference centiles for gestational ages 24-32 weeks. Early Hum Dev 1987; 15: $45-52$.

7 Pattinson R, Hope PL, Imhoff R, Manning N, Mannion V, Redman CWG. A case controlled study of obstetric and neonatal outcome in fetuses with absent end-diastolic flow in the umbilical artery. Am f Perinatol 1993; 10
133-6.

8 Cameron $\mathrm{AD}$, Nicholson SF, Nimrod CA, Harder JR Davies DM. Doppler waveforms in the fetal aorta and umbilical artery in patients with hypertension in pregnancy. Am F Obstet Gynecol 1988; 158: 339-45.

9 Meberg A. Transitory thrombocytopenia in newborn mice after intrauterine hypoxia. Pediatr Res 1980; 14: 1071-3. 